\title{
Parafilias: una revisión comparativa desde el DSM-5 y la CIE-10
}

Paraphilias: a comparative review from DSM-5 and CIE-10

\author{
Nahikari Sánchez Herrero ${ }^{1}$ \\ Rafael López Pérez ${ }^{2}$ \\ Antonio Domínguez-Muñoz ${ }^{3}$
}

(1) Centro Crimina - Universidad Miguel Hernández de Elche, Elche, España.

(2) Fundación Universitaria Behavior \& Law, Madrid, España.

(3) Instituto Nacional de la Seguridad Social, Madrid, España.

Email correspondencia:.nahikari.sanchez@me.com

\section{Resumen}

La comorbilidad de los delitos sexuales con parafilias hace que este tipo de trastornos tenga especial interés de cara a obtener información acerca del autor de un delito cuando este es aún desconocido y para establecer los correctos programas de intervención sobre este tipo de delincuentes. El estudio diagnóstico de las mismas parece, por tanto, ser clave para el posterior desarrollo de dichas intervenciones. Dado que en la actualidad tanto el Manual Diagnóstico y estadístico de los Trastornos Mentales -DSM-5- como la Clasificación Internacional de Enfermedades-CIE-son dos estándares de referencia para los profesionales de la salud, el presente artículo realiza una breve descripción comparativa de las parafilias recogidas en el DSM-5 y CIE-10. Posteriormente se revisan los tratamientos conductuales, cognitivo-conductuales, farmacológico y quirúrgico, para finalizar con las conclusiones obtenidas.

Palabras clave: delito sexual, delincuencia sexual, parafilia, pedofilia, fetichismo, exhibicionismo, voyeurismo, sadismo, masoquismo, frotteurismo.

\begin{abstract}
The comorbidity of sexual crimes with paraphilias makes this type of disorders especially interesting in order to obtain information about the perpetrator of a crime when it is still unknown and to establish the correct intervention programs on this type of offenders. The diagnostic study of the same seems, therefore, to be key for the subsequent development of these interventions. Since both the Diagnostic and Statistical Manual of Mental Disorders -DSM-5-and the International Classification of Diseases -ICD-are currently two reference standards for health professionals, this article provides a brief comparative description of the paraphilias collected in DSM-5 and ICD-10. Afterwards, the behavioral, cognitive-behavioral, pharmacological and surgical treatments are reviewed, in order to conclude with the conclusions obtained.
\end{abstract}

Keywords: sex offense, sexual delinquency, paraphilia, pedophilia, fetishism, exhibitionism, voyeurism, sadism, masochism, frotteurism,

Fecha de envío: 12/09/2018

Fecha de aceptación: 23/10/2018 


\section{INTRODUCCIÓN}

El tratamiento, evaluación y reincidencia de las personas que han cometido delitos sexuales es una cuestión que habitualmente causa alarma social, principalmente debido a la victimización que se produce, sobre todo, en los casos de violación y pederastia. En muchos delitos sexuales se comprueba que existe comorbilidad con comportamientos parafílicos, siendo su detección e intervención de gran interés para la disminución del riesgo y el mejor conocimiento del autor.

Por supuesto, no todos los delincuentes sexuales presentan una parafilia ni todos los individuos con una parafilia han llevado a cabo un delito sexual. Igual que no todos los delincuentes sexuales tienen las mismas necesidades de tratamiento (Kinsey, Pomeroy, y Martin, 1948; Marshall y Marshall, 2001; Marshall, 2007; Saleh y Guidry, 2003).

Parafilia se define actualmente en el diccionario de la Real Academia de la Lengua como "desviación sexual” y se refiere a casos en los que las personas presentan intereses sexuales atípicos. Desde el siglo XIX se han conocido como "perversiones sexuales" el incesto, la homosexualidad, la zoofilia, la pedofilia, el sadomasoquismo, el autoerotismo, el voyeurismo, el fetichismo, el travestismo, la coprofagia, las mutilaciones sexuales, etc. (Krafft-Ebing, 1886). Se recogían así manifestaciones sexuales diversas bajo un término amplio y heterogéneo. El término perversión sexual fue sustituido por el de parafilia en el año 1987 cuando, la American Psychiatric Association publica la versión revisada de su tercera edición del Manual Diagnóstico y estadístico de los Trastornos Mentales, el DSM-III-R, coincidente con la modificación clínica de la $9^{a}$ edición de la CIE (CIE9MC).

No obstante, el gran cambio acerca de que es "normal" o no en la conducta sexual se produjo a partir del llamado "Informe Kinsey", en realidad dos libros publicados alrededor del año 1950 (Kinsey, Pomeroy, y Martin, 1948 y
1953) que son aún motivo de controversia por sus implicaciones culturales, éticas y morales (Tarasco, 1997). Wardell Pomeroy, coautor de Kinsey, publicó posteriormente un artículo (Pomeroy, 1965) llamado ¿Qué es lo normal? en el que plantea "Sería más fácil borrar la palabra "normal" de nuestro vocabulario antes de contestar a la pregunta ¿Soy normal? Nuestra atención debe estar dirigida al ser humano individual y no al irrelevante, ilógico y psicológicamente dañino encasillamiento de las conductas sexuales en "normales o "anormales"”. Sin embargo, Pomeroy hace una propuesta muy interesante para abordar el problema de "lo normal" en Sexología, cuando propone la aplicación de cinco criterios principales para definir una conducta sexual como normal o anormal.

1. Estadístico: Una conducta sexual es normal cuando es practicada por la mayoría de la población. Influye el lugar, la época, la cultura y la clase social.

2. Filogenético o Biológico: Si corresponde con el comportamiento sexual de los primates superiores (o de los mamíferos...), es normal.

3. Moral: Los preceptos de una comunidad son variables en cada cultura y en diferentes épocas, pero suele haber un consenso, visible en usos, costumbres, creencias. Mantiene los valores individuales y colectivos.

4. Legal: El conjunto de normas escritas y sus sanciones para defender a las personas y su patrimonio o derechos también incluye lo sexual. Las leyes cambian y son diferentes en cada época y lugar, pero marcan el consenso social establecido.

5. Social: Las conductas socialmente dominantes que no perjudican a la sociedad o a sus miembros, pueden considerarse correctas o adecuadas a cada época.

Tabla 1. Revisión de conductas sexuales según criterios. Modificado de Pomeroy, 1965.

\begin{tabular}{|c|c|c|c|c|c|c|}
\hline Criterio & Masturbación & Homosexualidad & No Marital & S. Oral & Pedofilia & VIolación \\
\hline Estadístico & Normal & ¿? & Normal & Normal & Anormal & Anormal \\
\hline Etológico & Normal & Normal & Normal & Normal & ¿? & ¿? \\
\hline Moral & 508 & Anormal & Anormal & Normal & Anormal & Anormal \\
\hline Legal & Normal & Anormal & ¿? & Anormal & Anormal & Anormal \\
\hline Social & Normal & Normal & Normal & Normal & Anormal & Anormal \\
\hline
\end{tabular}


No extraña, por ello, que la tendencias actuales de los tratados internacionales -tanto el de la Asociación Americana de Psiquiatría (DSM-5) como el de la Organización Mundial de la Salud (CIE-10)- vayan hacia un modelo pretendidamente descriptivo, empirista y de corte epidemiológico, tratando de evitar juicios valorativos, ideológicos o morales, que no se corresponden con la categoría o concepto que designa cada trastorno.

El tratamiento de estos trastornos parafílicos ha evolucionado mucho con el paso de los años y con la investigación científica, disponiendo en la actualidad de fármacos que merece la pena describir, como el acetato de medroxiprogesterona (MPA), el acetato de ciproterona (CPA), los inhibidores selectivos de la recaptación de serotonina (ISRS), la triptorelina y la leuprorelina. (Brinjen, Nika y Berner, 2001; Gordon, 2008; Grubin y Haya, 2010; Kilmann, Sabalis, Gearing, Bukstel y Scovern, 1982; Marshall, 2007).

Con todo, parece que la conjunción de un tratamiento psicológico y un tratamiento farmacológico sería la mejor intervención con estos pacientes,(Gordon, 2008; Marshall, 2007; Thibaut, 2011) siempre teniendo en cuenta la necesidad de llevar a cabo intervenciones flexibles que puedan acomodarse al perfil de cada persona, a su conducta parafílica y a su situación penal (si han cometido un delito y se encuentran en prisión, en libertad condicional, disfrutando de salidas programadas, ya han terminado de cumplir con su pena o no han delinquido).

\section{DELINCUENCIA SEXUAL Y PARAFILIAS}

\section{A. Delincuencia sexual}

William L. Marshall (2001) define a los agresores sexuales como hombres (85-95\%) adultos o adolescentes y bastante parecidos al resto en sus características. Son, por lo tanto reflejo de la población general, observándose heterogeneidad entre las personas que llevan a cabo comportamientos sexuales de abuso y parafilias delictivas.

El impulso sexual en los mamíferos es innato. De este hecho no existe ninguna duda, pero la materialización de este impulso parece estar mediado por las condiciones ambientales y el aprendizaje del control de nuestro comportamiento. (Kinsey, Pomeroy y Martin, 1948; Marshall y Marshall, 2001). Así, los hombres deben aprender a mantener bajo control su tendencia innata a satisfacer sus propios deseos, en especial los relacionados con la agresión y la actividad sexual. Son los mismos sustratos neuronales los que actúan tanto sobre la agresión como sobre el comportamiento sexual, las conexiones neuronales parecen tener un gran parecido en estas áreas y los mismos esteroides que activan la agresión, activan el sexo (Moyer, 1976). El comportamiento sexual tiene como base un sistema endocrino que incluye diversos mediadores bioquímicos (Hucker y Bain, 1990) cuyo estudio es extenso y complicado, a pesar de que la investigación se ha centrado, casi de manera exclusiva, sobre la testosterona y su comportamiento. Los esteroides sexuales comienzan a activar nuestro comportamiento sexual y agresivo ya antes de la adolescencia, siendo esta la edad clave para comprender a canalizar los impulsos sexuales y violentos, además de desarrollar de manera correcta las tendencias sexuales permanentes (Contoni y Marshall, 1999; Marshall y Marshall, 2001). Cortoni y Marshall (2000) consideraron que los comportamientos sexuales de las primeras etapas de la adolescencia serían predictores de la conducta sexual adulta.

\section{B. Parafilias}

Las parafilias pueden definirse como la excitación sexual que se produce mediante el uso de determinados objetos, situaciones o el no consentimiento de otras personas. Medios de lograr una excitación sexual que, normalmente, se encuentran fuera del alcance de los intereses sexuales habituales. Money (1999) logró definir hasta 136 tipos diferentes de parafilias y Aggrawal (2009) planteó que existen, al menos, 547 categorías distintas.

Entre todas ellas, son ocho las que reclaman un mayor interés en este artículo, al estar incluidas dentro de DSM-5 y la CIE-10 de manera específica y ser susceptibles de causar los principales problemas en los individuos. El resto de los comportamientos parafílicos se incluirían dentro de las categorías residuales de parafilias.

El DSM-5 incluye un capítulo $\left(15^{\circ}\right)$ dedicado a los Trastornos Parafílicos, donde se establece que un interés sexual atípico no necesariamente es un desorden mental, dicho más claramente, que no toda parafilia (cuya descripción se realiza en el Criterio A) es un trastorno parafílico ya que éstos requieren, además el llamado Criterio B. Este puede corresponder a que los afectados sientan malestar psicológico (distress) causado por sus tendencias sexuales y no solamente como resultado de la desaprobación o repudio social. El otro criterio que permite el diagnóstico de Trastorno -y de especial interés en esta revisión- es tener un deseo o conducta sexual que implica malestar psicológico, lesiones o incluso la muerte de otra 
persona, o tener el deseo de una conducta sexual con personas que no quieren o no están impedidas de dar consentimiento. Estos últimos, debido a los daños a terceros, son tipificados como delitos sexuales.

Las parafilias pueden clasificarse (según DSM-5) en paidofilia, transvestismo, exhibicionismo, fetichismo, voyeurismo, masoquismo sexual, sadismo sexual, frotteurismo, otra parafilia especificada y no especificada (NOS). Finalmente no se ha incluido en la actual edición del Manual el trastorno coercitivo parafilico, que ya se había propuesto, con otro nombre para el DSM-III-R y había dado lugar a una viva polémica (Knight, 2010 y Wollert, 2011).

En la CIE-10, las parafilias están incluidas en el apartado F65 -Trastornos de la inclinación sexual- y en su mayoría coinciden con las del DSM-5. Así, trasvestismo (llamado fetichista, como en el DSM-IV-TR), exhibicionismo, voyeurismo (llamado también escoptofilia), paidofilia F65.1 a F65.4, respectivamente- y fetichismo (F65.0) se presentan también como parafilias específicas. Recoge un epígrafe (F65.5) dedicado al Sadomasoquismo, que incluye el sadismo y el masoquismo, como subepígrafes. En "Otros trastornos de la inclinación sexual" (F65.8) se incluirían los que en el DSM corresponden a "Otra parafilia especificada", que aquí incorpora el frotteurismo (F65.81) Habría un último epígrafe, llamado "Trastorno de la inclinación sexual sin especificación” (F65.9) que correspondería con la Parafilia NOS del DSM-5. Además, la CIE-10 reserva el código F65.6 "Trastornos múltiples de la inclinación sexual” para personas con más de una parafilia, sin que ninguna de ellas predomine. La combinación más frecuente sería fetichismo, transvestismo y sadomasoquismo.

Algunos de estos Trastornos parafílicos son considerados delitos sexuales, como el exhibicionismo, el frotteurismo, el voyeurismo o la pedofilia. Otros, como las conductas masoquistas o sádicas, están en la frontera de la legalidad, no siendo punibles si existe consentimiento de las personas adultas involucradas en dichas prácticas sexuales. Por último, los fetichismos, no son necesariamente ilegales aunque suelen estar mal vistos y pueden dar lugar a delitos menores, como hurtos de prendas de ropa, etc. Por todo ello, es fundamental el avance en las terapias e intervenciones con aquellas personas que padezcan algún tipo de trastorno parafílico, en especial en aquellos que están definidos como delito.

- Pedofilia o paidofilia. La pedofilia se puede definir como la excitación sexual que se produce en un individuo cuando tiene fantasías sexuales recurrentes e intensas, impulsos sexuales o comportamientos sexuales que conllevan actividad sexual con niños de 13 años o menos. La persona con la parafilia debe tener al menos 16 años y tener al menos 5 años más que la víctima. Este interés pedófilo resulta estable a lo largo de la vida del paciente, apareciendo por primera vez en la adolescencia.

Como se ha comentado al inicio, el conjunto de agresores sexuales es muy amplio y heterogéneo, y también lo es el de pedófilos, existiendo diferentes subtipos. Se puede distinguir a los pacientes que son atraídos por niños pre-púberes (pedófilos en sentido estricto) de aquellos que son atraídos por post-púberes (llamados también hebéfilos). También pueden ser menores pertenecientes a la familia o extraños, victimas femeninas o masculinas. Además, podemos observar un grupo que abusa de menores por otro tipo de razones como el poder, el control, o el sentido de derecho sobre el menor (incesto, principalmente). (Haya y Harkins, 2012; Marshall, 2007). La detección, la intervención y el tratamiento de los pacientes con cada uno de estos subtipos son muy diferentes, al igual que la valoración del riesgo de reincidencia.

- Transvestismo o Fetichismo transvestista. Se definiría como la excitación sexual, normalmente referida a hombres heterosexuales -aunque el DSM-5 ya incluye ambos sexos- que se produce ante la idea de verse a sí mismo vestido como mujer. No está considerado como ilícito y solamente es diagnosticado en el caso de que el paciente se angustie o afecte de tal manera que le impida llevar una vida normalizada.

- Exbibicionismo. Se trata de la excitación sexual que se produce cuando se lleva a cabo o se fantasea con la idea de exponer los genitales propios a otras personas sin el consentimiento de éstas. Suele aparecer durante la adolescencia, se trata de una conducta recurrente y se relaciona con el consumo de alcohol y drogas. Además, la revisión sistemática de la evidencia (Haya y Harkins, 2012) parece demostrar que tras una etapa más o menos prolongada de actividad exhibicionista, muchos de estos sujetos han llevado a cabo conductas de agresión sexual de contacto (violación, agresión o abuso sexual).

- Feticbismo. Corresponde a la excitación sexual en la que se ve implicado un objeto inanimado, entre los que estarían la ropa interior femenina o los zapatos. Este comportamiento sexual comienza en la pubertad a pesar de que la importancia del objeto estimulante pueda tener su origen en la infancia. No se trata de una 
conducta ilícita, siempre que no se hurten los objetos que producen esta excitación sexual. El DSM-5 incluye aquí el parcialismo, cuando la atención sexual está centrada exclusivamente en una parte del cuerpo no genital, por ejemplo, los pies.

- Voyeurismo. Consiste en la excitación sexual provocada por la visión de individuos desnudos, o con actividad sexual, siempre que estos no den su consentimiento para ser observados. Suele desarrollarse en la adolescencia. Se trata de un delito sin contacto que puede ser bastante victimizante.

- Masoquismo sexual. Implica la excitación sexual producida al ser humillado, golpeado, atado o mediante el padecimiento de dolor por algún medio. Suele desarrollarse a partir de los 20 años y en un amplio porcentaje dentro de estratos sociales elevados. A priori no se trata de un comportamiento ilícito, aunque se han producido casos en los que se ha causado un daño físico grave e incluso la muerte (Haya y Harkins, 2012; Harkins y Beech, 2012). El DSM-5 incluye aquí la asfixiofilia, cuando se usa la limitación o cese de la respiración durante la actividad sexual para incrementar la excitación y el orgasmo.

- Sadismo sexual. La excitación sexual aparece al infringir sufrimiento físico o psicológico (incluida la humillación) a otra persona. Los actos sádicos pueden aumentar en gravedad con el paso de los años, sobre todo en los sujetos con trastorno antisocial de la personalidad, llegando a producir daño físico grave e incluso la muerte. Se puede afirmar que no son muchos los sujetos que finalmente admiten la excitación sexual a través de la humillación y el sufrimiento de la víctima. (Beech, Fisher y Ward, 2005). Este diagnóstico resulta a menudo insuficiente para determinar la intervención a realizar, debiéndose tener en cuenta, además, el daño personal y social que se pudiese causar al sujeto al etiquetarlo como sádico sexual.

No tiene por qué tratarse en todo momento de un comportamiento ilícito, ya que puede que cierto comportamiento sádico sea consentido -e incluso deseadopor la pareja, aunque abandonaría esta condición si se llegase a causar daño físico grave o incluso la muerte. Históricamente, se ha diagnosticado a algunos violadores y asesinos -que no tienen por qué obtener placer sexual a través de sus delitos- como sádicos sexuales (MacCulloch, 2000), desvirtuando en algunos casos el diagnóstico y la posterior intervención.
- Frotteurismo. Implica la excitación sexual al tocar o frotarse contra una persona sin su consentimiento. También se incluyen las fantasías sexuales, impulsos sexuales o comportamientos relacionados. Suelen llevarse a cabo en lugares públicos con mucha gente, como autobuses, trenes o centros comerciales muy concurridos. Es una parafilia de alta frecuencia y normalmente es realizada por hombres, siendo las víctimas mujeres que son abusadas desde la parte de atrás de su cuerpo. Como dato de interés, en Japón son detenidos 4.000 frotteuristas masculinos al año por actos producidos en el transporte público. (Haya y Harkins, 2012). Esta parafilia tiene su comienzo en la adolescencia. No se trata de un comportamiento muy victimizante ya que muchas veces las víctimas ni siquiera se dan cuenta de lo que ocurre, sin embargo sí afecta a una gran cantidad de víctimas distintas y la evidencia científica parece demostrar que puede considerarse como una actividad precursora de delitos más graves, como la agresión o el abuso sexual (Freud y Seto, 1998).

- Otra Parafilia Especificada u Otros trastornos de la inclinación sexual. Dentro de este cajón de sastre encontramos, entre otros, la excitación sexual intensa y recurrente que implica la escatología telefónica (llamadas telefónicas obscenas), la necrofilia (cadáveres), la zoofilia (animales), la coprofilia (heces), la clismafilia (enemas) o la urofilia (orina).

Evidentemente, este sería un abordaje simplificado, a vista de pájaro, de estos trastornos. Cada uno de ellos -y no solo los recogidos individualmente en los tratadosprecisaría un análisis en mayor profundidad para intentar abarcar la complejidad del fenómeno, lo que excedería con mucho las posibilidades de este artículo. Valga como ejemplo, sin ánimo de exhaustividad, el tratado de los aspectos médico-legales de la necrofilia de Agrawal (2011), en el que recoge cinco clasificaciones previas de estas prácticas (Krafft-Ebing de 1886, Wulffen de 1910, Jones de 1931, Hirschfeld de 1956 y Rosman y Resnick de 1989). Describe entonces su propia propuesta de clasificación, con 10 clases de necrófilos; en la tabla siguiente se recogen los subtipos de la clase IX, "Necrófilos Homicidas", que incluye alguno de los más tristemente célebres asesinos en serie. 
Tabla 2. Varias subclases dentro de la Clase IX de Necrofília. Modificado de Agrawal, 2011.

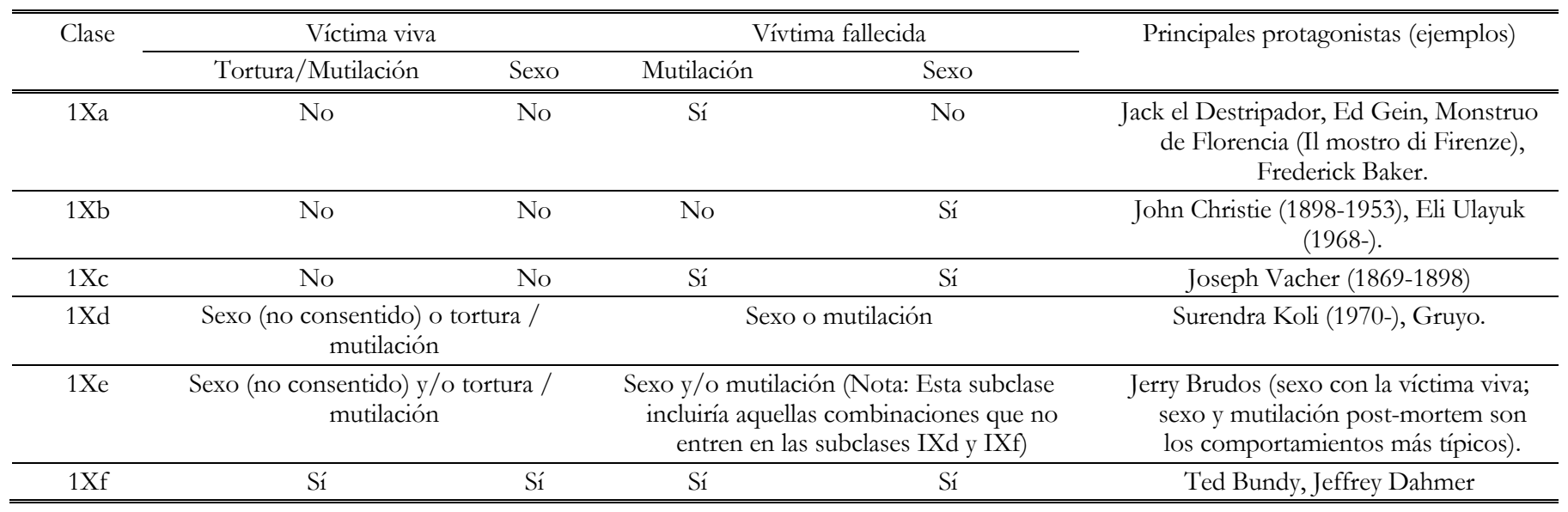

\section{TRATAMIEnTOS}

\section{A. Tratamiento conductual y cognitive-conductual}

El tratamiento sobre individuos con conductas parafílicas debe ser flexible y adaptarse a las necesidades que tenga el paciente y al ritmo de progreso que se lleve a cabo para resultar útil.

Hay que presentar una atención especial a la gran cantidad de diagnósticos comórbidos que se observan entre los pacientes parafílicos. Así, el 61,1\% de los pedófilos con víctimas femeninas tenían 3 o más parafilias adicionales, siendo un $54,2 \%$ de los pedófilos con víctimas masculinas y un $55,5 \%$ de los violadores los que presentaban 3 o más parafilias (Marshall, 2007). Entre las comorbilidades a las que hay que prestar una mayor atención está la posible presencia de psicopatía. Si el sujeto supera en 20 puntos la escala PCL-R, se debe considerar problemático el tratamiento. Éste deberá desarrollarse en un grupo y solamente podrá haber una persona con rasgos psicopáticos en el mismo para que, de esta manera, se pueda atenuar la tendencia oposicionista, disruptiva y manipuladora de estos sujetos. El narcisismo, así como el trastorno límite de la personalidad, son otras posibles comorbilidades que se deben tener presentes a la hora de establecer un tratamiento adecuado (Brinjen, Nika y Berner, 2001; Gordon y Haya, 2010; Kilmann, Sabalis, Gearing, Bukstel y Scovern, 1982).

En el tratamiento de las parafilias se han llevado a cabo principalmente intervenciones basadas en los principios de aprendizaje, de manera que su objetivo final consistía en modificar el interés sexual parafílico del sujeto y sustituirlo por otros. Estas intervenciones se denominan reacondicionamientos (Gordon y Haya, 2010; Haya y Harkins, 2012).
Para ello se han llevado a cabo técnicas como las siguientes:

\section{Técnicas conductuales}

- Terapia de aversión eléctrica. El objetivo de esta técnica es que cada vez que el paciente tenga un pensamiento relacionado con sus fantasías desviadas, sufra una descarga eléctrica en su brazo o pierna. Actualmente no están en uso por ser éticamente cuestionables.

- Terapia de aversión olfativa. La finalidad de esta terapia es relacionar las fantasías sexuales a evitar con una experiencia olfativa negativa, como el olor a carne podrida. Se realiza, normalmente, junto a otras técnicas.

- Terapia de aversión con uso de amoniaco. Similar a la aversión olfativa, en este caso se usan sales de amoniaco y su aspiración relacionada con el pensamiento sexual desviado.

- Sensibilización encubierta. Esta terapia se basa en forzar una relación entre el pensamiento o fantasía sexual desviada y un pensamiento o resultado desagradable. Se le pide al paciente que imagine una enfermedad tras el pensamiento desviado. Se lleva a cabo, normalmente, junto a otras técnicas (terapia de aversión olfativa, por ejemplo).

- Reacondicionamiento masturbatorio y Masturbación dirigida. Marques (2005) se refiere a esta técnica, asociando el orgasmo a fantasías no desviadas. Las investigaciones realizadas sobre este tratamiento no son muy alentadoras, ya que es un procedimiento muy difícil de controlar.

- Saciedad verbal. Esta técnica consiste en la verbalización repetida de fantasías sexuales desviadas hasta la 
reducción de la excitación sexual. El sujeto rápidamente termina de verbalizar sus fantasías pasando a otras que no ha llevado a la práctica, disminuyendo su interés y excitación sexual.

\section{Terapia cognitivo-conductual (TCC)}

Se trata de la principal terapia llevada a cabo con agresores sexuales, centrándose en la detección y eliminación de las distorsiones cognitivas. Se desarrollan habilidades para que los pacientes sean capaces de detectar los factores de riesgo y trabajar sobre ellos.

Se trata de realizar una evaluación sobre qué es lo que el paciente busca a través de la práctica de estas parafilias, qué intereses satisface, para proponerle las habilidades y actitudes necesarias para hacerlo de manera prosocial. Igualmente, se actúa sobre las"señales de alerta"en relación a sus ciclos de violencia sexual, precursores (pensamientos, situaciones o estados de ánimo detectados como previos al ciclo de abuso del paciente) con el objetivo de evitar recaídas mediante estrategias de evitación y afrontamiento. También se trabajan las competencias sexuales del sujeto, las habilidades sociales y funcionales y la autoestima. Se llevan a cabo programas de manejo de la ira y gestión del enfado, de relajación y actividades que promueven la empatía con la víctima.

No se debe llevar a cabo el mismo conjunto de módulos de tratamiento para todos los agresores sexuales con parafilias, independientemente de cómo estén funcionando. Hay que flexibilizar los programas para así mejorar la evaluación del riesgo de reincidencia.

\section{B. Tratamiento farmacológico y quirúrgico}

Son diferentes las terapias de corte biológico que se han llevado a cabo en los últimos años con el fin de reducir el riesgo de reincidencia en agresores sexuales y, aunque con resultados eficaces (siempre que se realicen bajo control médico), tienen elevados efectos secundarios. Se trata del tratamiento conocido popularmente como castración química y física. Algunos autores, como Graban y Haya (2010) aseguran que la castración física y química reducen el deseo sexual (libido) y el propio rendimiento sexual de algunos comportamientos parafílicos.

- Castración física. Técnicas como la orquiectomía o la neurocirugía estereotáxica se llevaron a cabo durante el siglo XX, aunque con muchas contraindicaciones éticas sobre todo derivadas del hecho de que no siempre se utilizaba con agresores sexuales de alto riesgo, ni eran proporcionales a los resultados obtenidos, siendo, en muchos casos técnicas que resultaron criminógenas.

- Castración química. Los fármacos antiandrogénicos actúan sobre los mecanismos biológicos que dependen de la testosterona. Algunos de estos fármacos son el acetato de ciproterona, la medroxiprogesterona y las gonadotropinas (GnRH) -leuprolida, goserelina y triptorelina- (Maletzky, Tolan y McFarland, 2006).

El CPA es un esteroide sintético, de forma similar a la progesterona que funciona inhibiendo el proceso de unión de la testosterona con sus receptores. Su uso está aceptado en más de 20 países. Se comercializa en comprimidos (50/200 mg / día) o como una inyección de liberación lenta (200/400 mg por semana o cada dos semanas). Es reversible tras uno o dos meses sin tratamiento (Grubin y Beech, 2010).

Las GnRH (triptorelina o leuprorelina) estimulan inicialmente la liberación de LH -hormona luteinizante-, dando lugar a un aumento de los niveles séricos de la testosterona, finalizando con una reducción de la LH y la testosterona a lo largo de las 2 a 4 semanas siguientes. La triptorelina se comercializa en dosis de $3 \mathrm{mg} / \mathrm{mes}$ y la leuprorelina como inyección intramuscular diaria o de liberación lenta (Prendky, 1997; Thibaut, 2011).

La finalidad de todos estos fármacos es reducir las concentraciones de testosterona a niveles prepuberales, consiguiendo que disminuyan las fantasías sexuales, la frecuencia de la eyaculación y que no puedan tener una erección (Maletzky, Tolan y McFarland, 2006)

Sus efectos secundarios son numerosos; diabetes, artrosis y osteoporosis, obesidad, eliminación del vello corporal, redistribución de la grasa (menos cintura y más cadera: feminización), crecimiento de mamas (ginecomastia), etc. (Haya y Harkins, 2012; Thibaut, 2011).

El principal problema asociado es que, ya que no pueden utilizar sus genitales para satisfacer sus impulsos violentos, usen otros objetos e instrumentos llegando a ser incluso más violentos -efecto criminógeno (Prendky, 1997)-. Por este motivo resulta fundamental el seguimiento médico y psicológico intensivo sobre este tipo de pacientes antes, durante y después del tratamiento para que se evalúen las consecuencias de los efectos adversos registrados y cómo son procesados por los pacientes, si los aceptan, si aparece frustración, ira, etc. (Lösel y Schumucker, 2005; Thibaut, 2011). 
En los últimos años se está trabajando, por este motivo, en la elaboración y síntesis de nuevos fármacos con menos efectos adversos, como el acetato de leuprolide. Después de una inyección en un área de músculo grande, leuprolide ejerce un efecto antilíbido por la inhibición de la secreción de LH y FSH -hormona folículoestimulante-. Su dosis terapéutica es de $7,5 \mathrm{mg} /$ mes o $22,5 \mathrm{mg} /$ cada 3 meses (Lösel y Schumucker, 2005; Maletzky, Tolan y McFarland, 2006; Thibaut, 2011).

\section{CONCLUSIONES}

A lo largo de este breve análisis se ha mostrado que, a pesar de que existen numerosos artículos dedicados a las parafilias, son pocos los estudios basados en la evidencia científica que aclaren su etiología, la eficacia de los tratamientos y de los que, por lo tanto, se pueda concluir con una prospectiva bien definida. Esta falta de estudios puede deberse a que los criterios del DSM-IV-TR y actualmente del DSM-5 y la CIE-10 han sido muy cuestionados, tachándolos más de legalistas y jurídicos, que de utilidad real para el diagnóstico y tratamiento de estos casos.

Quizá sería mucho más útil realizar una evaluación de las características de cada tipo de agresor sexual en dimensiones que vayan desde la normalidad hasta la problemática grave, sobre todo teniendo en cuenta la enorme heterogeneidad de este grupo de personas y comportamientos. Por ejemplo, ante la definición de Pedofilia, cabe preguntarse si la excitación sexual de un determinado adulto por niños de 14 años -y sus potenciales conductas asociadas- deben considerarse un comportamiento normal o como un trastorno psicológico que puede tratarse.

En la mayor parte de los estudios se establece que los comportamientos parafílicos están determinados en la infancia y al comienzo de la adolescencia, momentos clave del desarrollo de la bioquímica relacionada con los impulsos sexuales. Así, sería fundamental elaborar programas de prevención mediante la canalización de estos impulsos sexuales y violentos desde esas edades. De lo contrario, supondrán factores de riesgo que dispondrán a ser adultos con problemas de impulsos sexuales. La investigación debería centrarse en el estudio más exhaustivo de esta etapa vital y su desarrollo bioquímico.

Sería de sumo interés contar en el equipo técnico con herramientas específicas como el SVR-20, el STATIC-99 o el SVORAG en adultos y el ERASOR en adolescentes con conductas de agresión sexual. De esta manera podremos evaluar los factores de riesgo y de protección existentes en el paciente y realizar la intervención y el tratamiento de una forma mucho más eficaz, coordinada, individualizada y, por lo tanto, eficiente.

En relación con los tratamientos de los trastornos parafílicos delincuenciales se debe tener presente su elevada comorbilidad con otros trastornos. Se observa que una combinación de tratamiento cognitivo-conductual junto con farmacoterapia parece resultar lo más adecuado, obteniéndose un menor riesgo de reincidencia.

Ambos elementos del tratamiento -TCC y farmacoterapia- deben realizarse bajo un estricto seguimiento médico y psicológico y en un periodo de tiempo amplio, ya que los efectos adversos derivados de la farmacoterapia pudieran ser graves, convirtiendo el tratamiento en criminógeno. Además, debemos poner especial cuidado en la discreción requerida -dado lo delicado de estas cuestiones- y tener en cuenta el daño que, personal y socialmente, podemos hacer a un sujeto al etiquetarlo con un trastorno parafílico.

\section{REFERENCIAS}

Aggrawal, A. (2009) Forensic and medico-legal aspects of sexual crimes and unusual sexual practices. Boca Ratón: Taylor and Francis.

Aggrawal, A. (2011) Necrophilia: forensic and medico-legal aspects. Boca Ratón: CRC Press.

American Psychiatric Association (APA). (1988) Manual Diagnóstico y Estadístico de los Trastornos Mentales (4 ${ }^{\mathrm{a}}$ ed. revisada) (DSM-III-R). Barcelona: Masson.

American Psychiatric Association (APA). (2002) Manual Diagnóstico y Estadístico de los Trastornos Mentales ( $3^{\mathrm{a}}$ ed. revisada) (DSM-IV-TR). Barcelona: Masson.

American Psychiatric Association (APA). (2014) Manual Diagnóstico y Estadístico de los Trastornos Mentales (5 ${ }^{\mathrm{a}}$ ed.) (DSM-5). Madrid: Panamericana.

Beech, A., Fisher, D. y Ward, T. (2005). Sexual Murderer's Implicit Theories. Journal of Interpersonal Violence, 20, 1366-1389.

Brinjen, P.; Nika, E. y Berner, W. (2001) Treatment of paraphilias with luteinizing hormone-releasing hormone agonist. Journal os sex and marital therapy, 27, 45-55.

Cortoni, F.A. y Marshall, W.L. (2000). Sex as a coping strategy and its relationship to juvenile sexual history and intimacy in sexual offenders, en prensa.

Freud, K. y Seto, M.C. (1998). Archives of sexual behavior, 27, 433-443.

Gordon, H. (2008). Editorial. The treatment of paraphilias: An historical perspective. Criminal behavior and Mental Health, 18, 79-87.

Grubin, D. y Beech, A. (2010). Chemical castration for sex offenders. British Medical Journal, 340. 
Grubin, D. y Haya, A.R. (2010) Chemical castration for sex offenders: Doctors should avoid becoming agents of social control. British Medical Journal, 340, c74.

Hare, R.D. (1991). Manual for The Hare Psychopathy Checklist-Revised. Toronto: Multi-Health Systems.

Harkins, L. y Beech, A.R. (2012). Paraphilias and sexual offending. Handbook of evidence-based practice in clinical psychology, 2, 646-678.

Haya, A.R. y Harkins, L. (2012). DSM-IV paraphilia: Descriptions, demographics and treatment interventions. Aggression and Violent Behavior, 17, 527-539.

Hucker, S.J. (2008). Sexual masochism: Assessment and treatment. In D.R. Laws and W. O’Donohue (Eds.) Sexual deviance: theory, assessment and treatment. New York: Guilford.

Hucker, S.J. y Bain, J. (1990). Androgenic hormones and sexual assault, en W.L. Marshall, D.R. Laws y H.E. Barbarize (Eds.), Handbook of sexual assault: Issues, theories, and treatment of the offender, New York, Plenum Press, pp. 93-102.

Kilmann, P.R.; Sabalis, R.F.; Gearing, M.L.; Bukstel, L.H. y Scovern, A.W. (1982) The treatment of sexual Paraphilias: a review of the outcome research. The Journal of Sex Research, 18(3), 193-252.

Kinsey, A.C.; Pomeroy, W.B.; Martín, C.E. (1948) Sexual behavior in the human male. Philadelphia: W.B. Saunders.

Kinsey, A.C.; Pomeroy, W.B.; Martín, C.E. (1953). Sexual behavior in the buman female. Philadelphia: W.B. Saunders.

Knight RA. (2010) Is a diagnostic category for paraphilic coercive disorder defensible? Arch Sex Behav, 39(2), 419-26.

Krafft-Ebing, RF. (1886). Psychopathia Sexualis (1st Ed.). Philadelphia: FA Davis.

Lösel, F. y Schumucker, M. (2005). The effectiveness of treatment for sexual offenders: A comprehensive meta-analysis. Journal of Experimental Criminology, 1, 117-146

MacCulloch, M, Gray, N, y Watt, A. (2000). Brittain's Sadistic Murderer Syndrome reconsidered: an associative account of the aetiology of sadistic sexual fantasy. The Journal of Forensic Psychiatry, 11(2), 401-418.

Maletzky, B.M., Tolan, A. y McFarland, B. (2006). The Oregon DepoProvera Program: A Five- Year Follow-UP. Sexual Abuse, 18, 303-316

Marques, J. K., Wiederanders, M., Day, D. M., Nelson, C., y van Ommeren, A. (2005). Effects of a relapse prevention program on sexual recidivism: Final results from California's sex offender treatment and evaluation project (SOTEP). Sexual Abuse: A Journal of
Research and Treatment, 17(1), 79-107

Marshall, L.E. y Marshall W.L. (2001) Excessive desire disorder among sexual offenders: The development of a research Project. Sexual Addiction and Compulsivity: The Journal of Treatment and Prevention, 8, 301307

Marshall, W.L. (2007) Diagnostic issues, multiples paraphilias, and comorbid disorders in sexual ofenders: Their incidence and tratment. Aggression and Violent Behavior, 12, 16-35

Michael, B. y Frances, A. (2008) issues for DSM-V: Unintended consequences of small changes. The case of paraphilias. The American Journal of Psychiatry. 165(10), 1240-1241

Money, J. (1999) The lovemap guidebook: A definitive statement. New York: Continuum.

Organización Mundial de la Salud. (1992) CIE 10: Décima Revisión de la Clasificación Internacional de Las Enfermedades. Trastornos Mentales y del Comportamiento: Descripciones Clínicas y pautas para el Diagnóstico. Madrid: Meditor

Pomeroy, W.B. (1965). 'What Is Normal? Hef's Philosophy: Playboy and Revolution from 1965-1975. Playboy, 12. En https://forthearticles.omeka.net/items/show/11

Prendky, R.A. (1997). Arousal reduction in sexual offenders: A review of antisndrogen interventions. Sex Abuse, 9, 335-347

Rosman, J.P. y Resnick, P.J. (1989). Sexual attraction to corpses: A psychiatric review of necrophilia. The Bulletin of the American Academy of Psychiatry and the Law, 17(2), 153

Saleh, F.M. y Guidry, L.L. (2003) Psychosocial and biological treatment considerations for the paraphilic and nonparaphilic sex ofender. The Journal of American Academy of Psychiatry and the Law, 31(4), 486-493

Saleh, F.M.; Martin Malin, H.; Grudzinskas, A.J. y Vitacco M.J. (2010) Paraphilias with co-morbid psychopathy: the clinical and legal significance to sex offender assessments. Behavioral Sciences and Law, 28, 211-223.

Tarasco Michel, M. (1997) Consideraciones sobre la influencia del reporte Kinsey. Cuadernos de bioética, 32(8), 1385-1397.

Thibaut, F. (2011). Pharmacological treatment of sex offenders. Sexologies, 20, 166-168

Wollert, R. (2011) Paraphilic coercive disorder does not belong in DSM5 for statistical, historical, conceptual and practical reasons. Archive Sexual Behavior, 40, 1097-1098. 